\title{
CT features and differential diagnosis of primary pulmonary mucoepidermoid carcinoma and pulmonary adenoid cystic carcinoma
}

\author{
Xian Li $^{1}$, Wei Yi ${ }^{2}$, Qingsi Zeng ${ }^{1}$ \\ ${ }^{1}$ Department of Radiology, ${ }^{2}$ Department of Radiotherapy, First Affiliated Hospital of Guangzhou Medical University, Guangzhou, Guangdong \\ 510120, China \\ Contributions: (I) Conception and design: Q Zeng; (II) Administrative support: Q Zeng; (III) Provision of study materials or patients: W Yi; \\ (IV) Collection and assembly of data: W Yi; (V) Data analysis and interpretation: X Li; (VI) Manuscript writing: All authors; (VII) Final approval of \\ manuscript: All authors. \\ Correspondence to: Qingsi Zeng, MD. Department of Radiology, First Affiliated Hospital of Guangzhou Medical University, Guangzhou, Yanjiang \\ Road 151, Guangdong 510120, China. Email: gzlixian2011@163.com.
}

Background: The differential diagnosis of primary pulmonary mucoepidermoid carcinoma (PMEC) and pulmonary adenoid cystic carcinoma (PACC) is difficult, because both tumors could be similar in terms of certain characteristics on CT.

Methods: The CT findings from 24 cases of PMEC and 30 cases of PACC were retrospectively analyzed. According to the position of the lesion in airway, we divided these cases into three types: central, hilar, and peripheral.

Results: In PMEC, there were 7 cases of central type, 14 cases of hilar type, and 3 cases of peripheral type. And, 57.1\% PMEC cases of the hilar type were accompanied by distal bronchial dilatation with mucoid impaction. Patchy areas of low density were observed in $79.2 \%$ cases of PMEC. The solid part of most lesions showed moderate $(37.5 \%)$ or severe enhancement (45.8\%). However, in PACC, there were 24 cases of central type, 3 cases of hilar type, and 3 cases of peripheral type. PACC had more cases of central type than PMEC. Moreover, longitudinal extent greater than $3 \mathrm{~cm}$ was observed in $62.5 \%$ PACC cases of the central type, while infiltration of the luminal perimeter more than $1 / 2$ perimeter was observed in $95.8 \%$ PACC cases of the central type. Patchy areas of low density were observed in 26.7\% cases of PMEC. In PACC cases, the solid part of $76.7 \%$ lesions showed slight enhancement. Cavities could be observed in PMEC, but not in PACC.

Conclusions: PMEC and PACC have different CT features in various airway locations. PMEC is usually the hilar type, accompanied by distal bronchial dilatation with mucoid impaction. However, PACC is usually the central type, with longitudinal extent greater than $3 \mathrm{~cm}$ and infiltration of the luminal wall more than $1 / 2$ perimeter. Patchy areas of low density and moderate or severe enhancement are more prominent in PMEC. However, slight enhancement is more common in PACC.

Keywords: Pulmonary mucoepidermoid carcinoma (PMEC); pulmonary adenoid cystic carcinoma (PACC); tomography; X-ray computed; identification

Submitted Mar 15, 2018. Accepted for publication Nov 14, 2018.

doi: $10.21037 /$ jtd.2018.11.71

View this article at: http://dx.doi.org/10.21037/jtd.2018.11.71 


\section{Introduction}

Mucoepidermoid carcinoma and adenoid cystic carcinoma are common in the parotid, submandibular, and other small salivary glands (1). Pulmonary mucoepidermoid carcinoma (PMEC) and pulmonary adenoid cystic carcinoma (PACC) are usually considered to originate from the bronchial glands of minor salivary gland-type lining the tracheobronchial tree $(2,3)$. The bronchial glands are located in the submucosa of large airways, similar to the head and neck salivary glands. Both two diseases are rare salivary gland-type tumors of lung, accounting for fewer than $2 \%$ of all lung cancers (4). In salivary gland-type tumors of the lung, the most common histological type is PACC, comprising about two-thirds of the cases, with PMEC comprising about a third (5). The purpose of this paper is to realize and summary the CT features of PMEC and PACC. It is important and helpful to take differential diagnosis of two diseases, because PMEC and PACC both originate from salivary glands in the tracheobronchial tree and have similar imaging signs. To decrease the diagnostic errors and improve diagnosis, the CT features of PMEC and PACC were analyzed according to various airway locations.

\section{Methods}

\section{Subjects}

We analyzed 24 cases of PMEC and 30 cases of PACC from January 2011 to December 2017. In case of PMEC, there were 13 male and 11 female patients, aged 5-72 years (median, 30 years). In case of PACC, there were 9 male and 21 female patients, aged $21-81$ years (median, 48 years). The clinical presentation of both diseases included cough, phlegm, hemoptysis, fever, back pain, and shortness of breath.

\section{Instruments and methods}

The Siemens Definition AS 128 multi-detector-row computed tomography was used to scan the plain and enhanced CT images. The tube voltage was $120 \mathrm{kV}$, with thickness of $2 \mathrm{~mm}$ and FOV of $250 \mathrm{~mm} \times 250 \mathrm{~mm}$. Iopromide injection $(1.20 \mathrm{~mL} / \mathrm{kg})$ was used as contrast, with a concentration of $370 \mathrm{mg} / \mathrm{mL}$ and flow rate of $4.30 \mathrm{~mL} / \mathrm{s}$. Then, $30 \mathrm{~mL}$ saline was administered at the same flow rate.

\section{Image analysis}

Location, size, edge, density, enhancement, necrosis, and calcification were observed and analyzed in the lesions. The infiltration of surrounding tissue, presence of pleural effusion, and enlargement of hilar or mediastinal lymph nodes were detected as well.

\section{Operation and pathological examination}

Tissues from lesions were obtained by surgical resection or biopsy. When the lesion had extensive infiltration unsuited for surgical resection, a biopsy was taken. Both lesions from surgical resection and biopsy were examined by pathological section and HE staining. All cases were clearly identified by two pathologists, who referred to the clinical and imaging data.

\section{Results}

\section{PMEC}

\section{Classification according to location}

Twenty-four cases of PMEC were divided into three groups based on the location of the lesion in the airway. The analysis was based on previous studies (6,7) and analysis of CT images. Seven cases were classified as the central type, which were located in the trachea or the main bronchi. The hilar type included 14 cases, which were located in the lobar or segmental bronchi (including right middle bronchus). Three cases in the small bronchi were the peripheral type.

\section{Configuration of lesion, size of lesion, and accompanying signs}

In the 7 PMEC cases of the central type, the diameter of the lesions was all less than $3.0 \mathrm{~cm}$. These lesions were mainly intraluminal nodules (Figure 1). Only 1 case vegetated outside the lumen. The tumor infiltration of perimeter was less than $1 / 2$ perimeter of the luminal wall in all cases. On coronal image, the longitudinal extents were all less than $3.0 \mathrm{~cm}$. One case had left complete atelectasis in the left main bronchus.

Fourteen PMEC cases of hilar type had nodules in the lobar or segmental bronchi (including right middle bronchus). Thirteen cases had diameters less than $3.0 \mathrm{~cm}$, of which eight cases $(57.1 \%)$ were accompanied by distal bronchial dilatation with mucoid impaction (Figure 2). Only 1 case showed a mass measuring more than $3 \mathrm{~cm}$ in the right upper bronchus, accompanied by complete atelectasis of the right upper lung.

Three PMEC cases of the peripheral type were all masses 

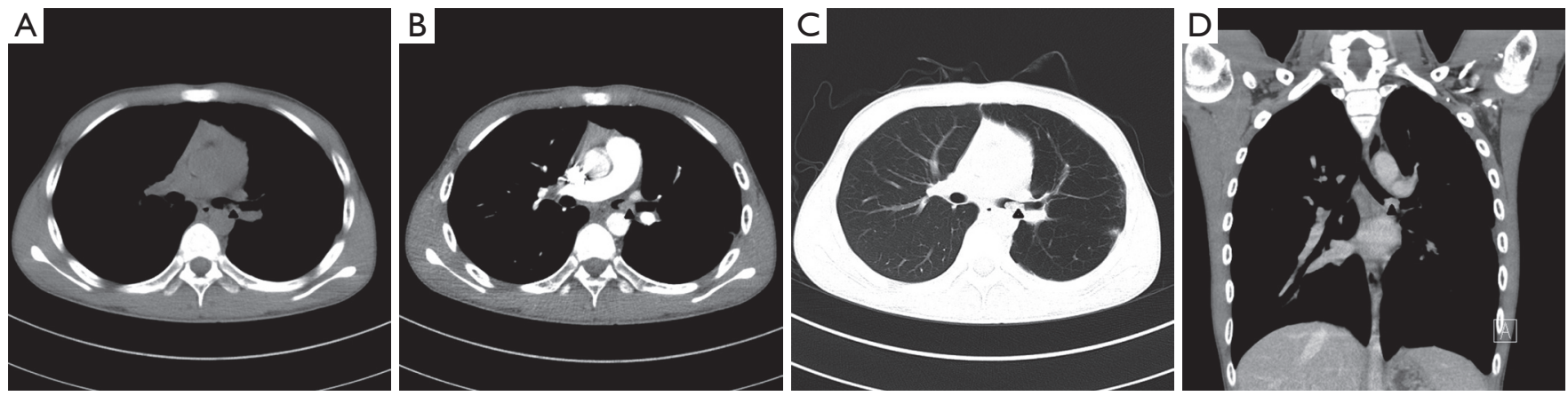

Figure 1 PMEC of central type in left main bronchi. (A) Plain transverse image of mediastinal window; (B) enhanced transverse image of mediastinal window; (C) transverse image of lung window; (D) enhanced coronal image of mediastinal window. Nodule in lumen of left main bronchi was close to the back and subjacent wall. In enhanced image, the solid part of lesion had severe enhancement and patchy area of low density was observed inside the lesion. The black allow head was on behalf of the lesion. PMEC, pulmonary mucoepidermoid carcinoma.
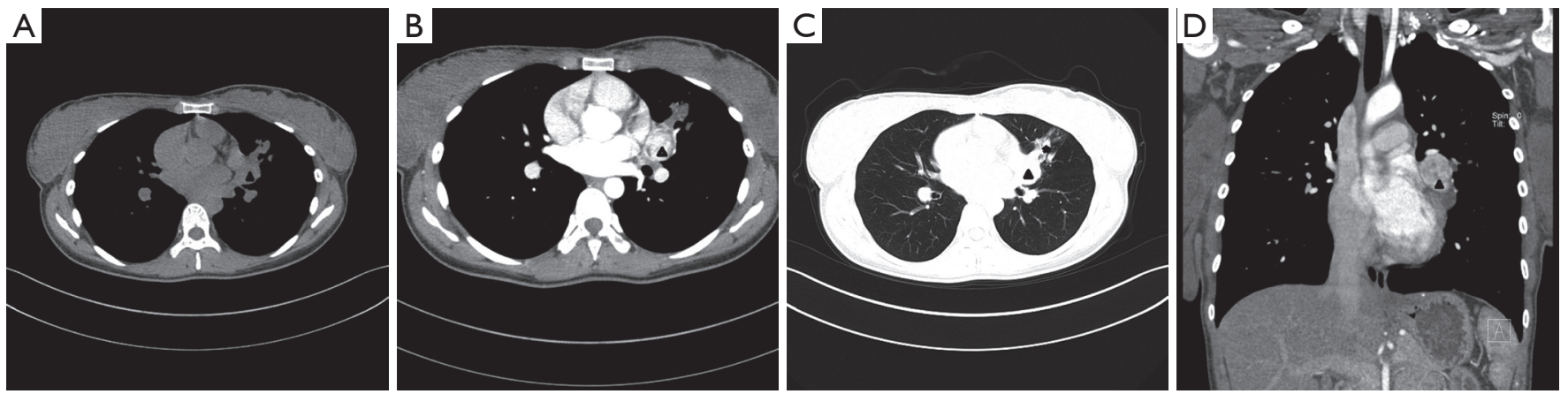

Figure 2 PMEC of hilar type in lingual segment of left upper lung. (A) Plain transverse image of mediastinal window; (B) enhanced transverse image of mediastinal window; (C) transverse image of lung window; (D) enhanced coronal image of mediastinal window. Intraluminal lesion in lingual segment of left upper lung was in left hilar area, which was accompanied by distal bronchial dilatation with mucoid impaction. Patchy area of low density was observed inside the lesion. The solid part of lesion had severe enhancement. The black allow head represented the intraluminal lesion in lingual segment of left upper lung, and the black star represented distal bronchial dilatation with mucoid impaction. PMEC, pulmonary mucoepidermoid carcinoma.

(Figure 3), whose diameter was more than $3 \mathrm{~cm}$. These lesions originated from small bronchi and away from large airways. In the peripheral type, no case was accompanied by distal pulmonary atelectasis or distal bronchial dilatation with mucoid impaction.

\section{Lesion density and enhancement}

In the 7 PMEC cases of central type, 4 cases had patchy areas of low density inside the lesion. Twelve cases had patchy areas of low density in the 14 cases of hilar type. No cavity was seen in any lesion of the central and hilar types. Moreover, 3 cases of peripheral type had heterogeneous density, of which 2 cases had large cavities. Therefore, $79.2 \%$ cases (19/24) had patchy areas of low density. Calcification was observed in 2 cases of hilar type.

The degree of enhancement was classified as slight enhancement, moderate enhancement, and severe enhancement. Comparing the enhanced and plain CT values, an increase less than $20 \mathrm{HU}$ was considered slight enhancement. Increase of CT value less than $40 \mathrm{HU}$ and more than $20 \mathrm{HU}$ was considered moderate enhancement. Severe enhancement was defined as an increase of CT value more than $40 \mathrm{HU}$.

Nine PMEC cases (37.5\%) had moderate enhancement of the solid part, and 11 cases $(45.8 \%)$ had severe enhancement of the solid part. Only 4 cases had slight enhancement of the solid part. 

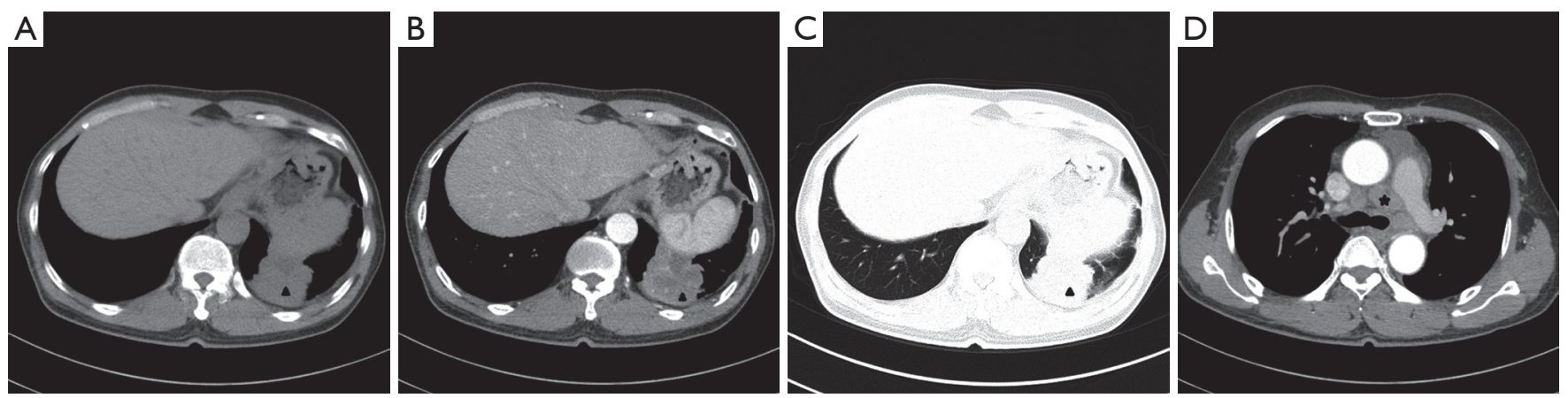

Figure 3 PMEC of peripheral type in left lower lung. (A) Plain transverse image of mediastinal window; (B) enhanced transverse image of mediastinal window; (C) transverse image of lung window; (D) enhanced transverse image of mediastinal window. A mass with patchy area of low density was in left lower lung, which was accompanied by metastasis of hilar and mediastinal lymph nodes. The black allow head represented the lesion of peripheral type in left lower lung, and the black star represented enlarged lymph nodes. PMEC, pulmonary mucoepidermoid carcinoma.

\section{Adjacent infiltration of lesion and metastasis of lymph nodes \\ Of the 24 PMEC cases, infiltration of surrounding fat interval was discovered in only 2 cases; of which, one case was the central type, and the other was the hilar type. Pleural effusion and pleural thickness were not detected. Only 3 cases had metastasis of hilar and/or mediastinal lymph nodes.}

\section{Pathology}

Lesions of 24 PMEC cases were taken by surgical resection or biopsy, including 19 cases of surgical resection and 5 cases of biopsy. PMEC were composed of mucous cells, epidermal cells, and intermediate cells. Mucous cells were polygonal or high column-shaped. Much mucus was seen inside and outside the mucous cells. According to their histological characteristics, PMEC was divided into lowgrade and high-grade malignancy (8). High-grade PMEC was characterized by necrosis, nuclear pleomorphism, frequent mitoses, and a solid or nested pattern of growth for the intermediate or squamous cells. Low-grade PMEC usually demonstrated bland cytological features, few mitoses, frequent cystic components, and mucous cells. There were 13 cases of low-grade malignancies, including 5 cases of central type and 8 cases of hilar type. The other 11 cases were high-grade malignancies, including 2 cases of central type, 8 cases of hilar type, and 3 cases of peripheral type. Patchy areas of low density inside the lesion were cystic degeneration in cases of low-grade malignancy, but were necrosis in cases of high-grade malignancy.

\section{PACC}

\section{Classification according to location}

PACC were also divided into three groups according to the lesion's location in the airway. The central type included 24 cases, which were located in the trachea (17 cases) and the main bronchus (7 cases). The hilar type included 3 cases, and the peripheral type also included 3 cases.

\section{Configuration, size, and accompanying signs}

In 24 PACC cases of the central type, the luminal wall of the trachea or main bronchus was thickened. The thickened wall could be nodular or heterogeneous in the trachea (Figure 4) or the main bronchus. Vegetations outside the lumen were observed in $75 \%$ cases (18/24). All cases had a diameter less than $3 \mathrm{~cm}$. However, the longitudinal extent of $62.5 \%$ cases $(15 / 24)$ was greater than $3 \mathrm{~cm}$ on the coronal image, and $29.2 \%$ cases $(7 / 24)$ infiltrated the inferior bronchus. Infiltration could be observed in the adjacent luminal wall of the lesions, throughout the luminal wall. Twenty-three cases (95.8\%) showed infiltration of the perimeter more than $1 / 2$ perimeter of luminal wall. Complete atelectasis of the left or right lung was found in only 3 cases, originating from the left or right main bronchus.

Three PACC cases of hilar type were both masses, accompanied by lobar atelectasis (Figure 5). And there were also 3 PACC cases of peripheral type, which were masses or nodules originating from small bronchi (Figure 6). Pulmonary atelectasis or obstructive pneumonia was not 

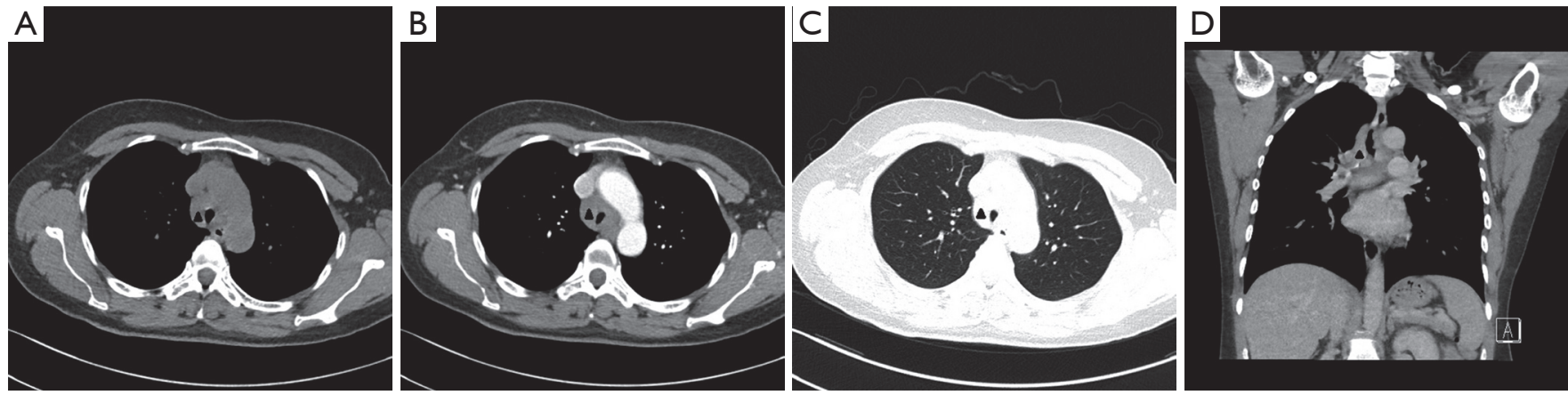

Figure 4 PACC of central type in trachea. (A) Plain transverse image of mediastinal window; (B) enhanced transverse image of mediastinal window; (C) transverse image of lung window; (D) enhanced coronal image of mediastinal window. Infiltration of the tracheal luminal perimeter was more than $3 / 4$ perimeter, and the longitudinal extent was greater than $3 \mathrm{~cm}$ in coronal image. In enhanced image, the lesion had slight enhancement. The black allow head was on behalf of the lesion. PACC, pulmonary adenoid cystic carcinoma.
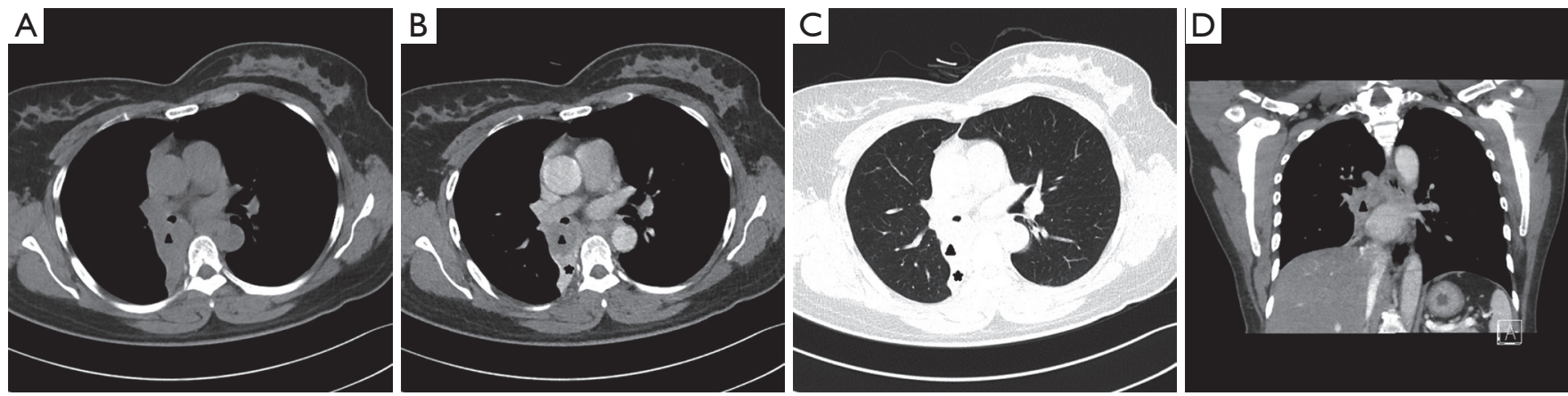

Figure 5 PACC of hilar type in back segment of right lower lung. (A) Plain transverse image of mediastinal window; (B) enhanced transverse image of mediastinal window; (C) transverse image of lung window; (D) enhanced coronal image of mediastinal window. Intraluminal lesion in back segment of right lower lung was in right hilar area, which was accompanied by distal pulmonary atelectasis. The lesion had slight enhancement, which was different from distal pulmonary atelectasis. The black allow head represented the intraluminal lesion, and the black star represented distal pulmonary atelectasis. PACC, pulmonary adenoid cystic carcinoma.
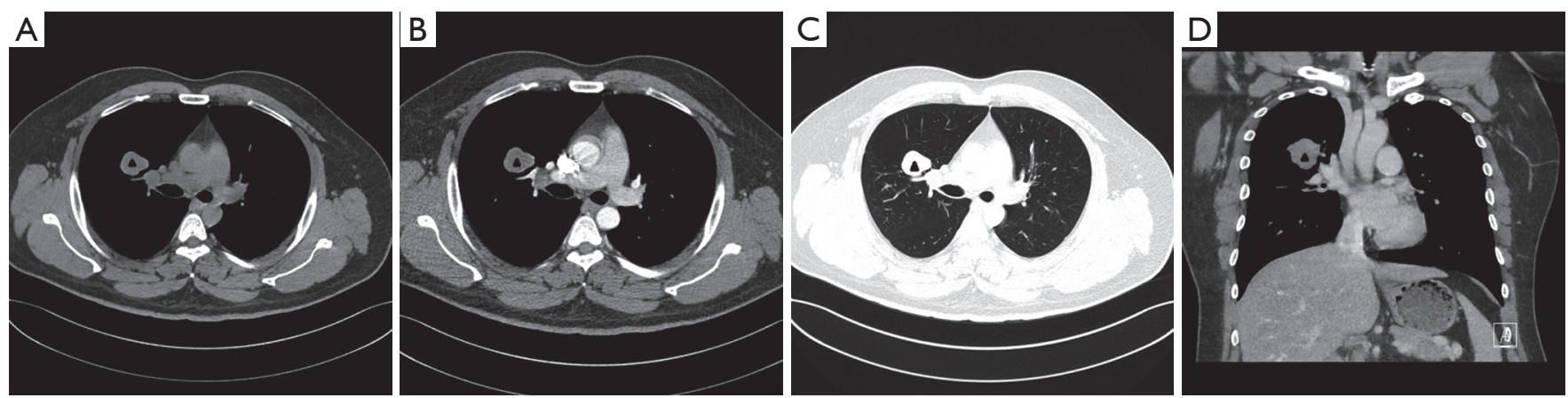

Figure 6 PACC of peripheral type in right upper lung. (A) Plain transverse image of mediastinal window; (B) enhanced transverse image of mediastinal window; (C) transverse image of lung window; (D) enhanced transverse image of mediastinal window. A mass without patchy area of low density was in right upper lung. The lesion had slight enhancement. The black allow head represented the lesion of peripheral type. PACC, pulmonary adenoid cystic carcinoma. 
detected in the peripheral type.

\section{Density and enhancement}

The density of PACC lesions was usually homogeneous. Only 8 cases (26.7\%) of the central type had patchy areas of low density inside the lesion. Calcification and cavities were not seen in any PACC case.

Twenty-three cases $(76.7 \%)$ showed slight enhancement of the solid part. Only 6 cases $(20 \%)$ had moderate enhancement and 1 case (3.3\%) had severe enhancement of the solid part.

\section{Infiltration of the lesion and metastasis to lymph nodes} Of the 30 PACC cases, 19 cases of central or hilar type showed infiltration of the mediastinal fat, even the thyroid or the esophagus. Pleural effusion and pleural thickening were not detected. Eleven cases had metastases of hilar and/ or mediastinal lymph nodes.

\section{Pathology}

Lesions of 30 PACC cases were taken by surgical resection or biopsy, including 23 cases of surgical resection and 7 cases of biopsy. PACC are composed of ductal epithelial cells and mutated myoepithelial cells. Ductal epithelial cells were cubic or oval, uniform in size. The cytoplasm of epithelial cells was scant, usually transparent. The round or ovoid cell nucleus was large and heavily stained. Mutated myoepithelial cells were flat and irregular. Under the light microscope, two cells were arranged in solid, tubular, or cribriform structures. Mucus was seen in the glandular lumen or cribriform holes. Mucous degeneration could also be observed in the interstitial structure of tumor. Tumor necrosis and nuclear fission were uncommon.

\section{Discussion}

\section{Position of the lesion}

PMEC is usually located in a lobar or segmental bronchi (including the right middle bronchus). The lesion in the main bronchi and trachea are relatively less often involved, even less so in small bronchi. Tae also reported that $10 \%$ of lesions were in the main bronchi and trachea, $75 \%$ were located in lobar or segmental bronchi, and $15 \%$ were in small bronchi (6). In a Chinese series, Jun-Jie Xi reported that PMEC could be found at all levels of the airway, especially the lobar or segmental bronchi (including the right middle bronchus) (7). Additionally, this disease was found relatively less often in the main bronchi and trachea, and rarely in small bronchi. We could find a similar distribution in our study. The hilar type was the most common of the three groups, and the peripheral type was the least common. On the contrary, PACC were mainly of the central type in the study. Some studies also reported PACC usually located in the trachea or the main bronchi $(9,10)$. Our study showed similar results. The cases of PACC were mainly central type, located in the trachea and the main bronchi.

\section{Configuration, size, and accompanying signs}

\section{The central type}

In another study (11), the central type of PMEC was characterized by limited nodules. The tumors appearance included a circumferential or elevated soft tissue nodule with either a smooth, lobulated, or polypoid margin (12). In our study, the central type of PMEC also showed nodules, with perimeter infiltration less than $1 / 2$ perimeter of luminal wall and longitudinal extent less than $3.0 \mathrm{~cm}$. However, in PACC, the central type was the most common, with heterogeneous or homogeneous thickened walls. The most central type of PACC had wide longitudinal extent greater than $3 \mathrm{~cm}$ and perimeter infiltration greater than $1 / 2$ perimeter of luminal wall. Kwak et al. also reported that the luminal wall could be nodular or of uniform thickness, with extension to more than $180^{\circ}$ of the luminal perimeter (13).

Therefore, there is substantial difference between the central type of PMEC and PACC. It should be noted that submucosal spread is a feature of primary adenoid cystic carcinoma (10). However, in some situations, the lesions of PMEC and PACC could all be expressed as limited nodules. Nevertheless, it is difficult to distinguish PMEC from PACC when the lesions of PACC are nodules with limited perimeter infiltration.

\section{The hilar type}

PMEC of the hilar type usually manifested with nodules inside the lobar or segmental lumen, often accompanied by distal bronchial dilatation with mucoid impaction (14). In our study, all cases of hilar type had nodules inside the lobar or segmental lumen, and about half of the PMEC cases were accompanied by distal bronchial dilatation with mucoid impaction. Only a small number of PACC were accompanied by lobar atelectasis, but not distal bronchial dilatation with mucoid impaction. Therefore, nodules in the 
lobar or segmental lumen and the distal bronchial dilatation with mucoid impaction were characteristic features of the hilar type in PMEC.

\section{The peripheral type}

The peripheral type was the least found type both in PMEC and PACC. These lesions were all masses, originating from small bronchi. When PMEC and PACC of peripheral type are both mass, it is difficult to distinguish between the two diseases. However, it should be noted that PMEC could have large cavities inside the lesion, but not PACC.

\section{Density}

In our study, patchy areas of low density were usually observed in PMEC. However, patchy areas of low density were not often found in PACC. Cystic degeneration in cases with low-grade malignancy or necrosis in cases with high-grade malignancy was more common in PMEC than in PACC (15). PACC lesion had few cystic degeneration or necrosis according to the pathological result. As the necrotic material could be eliminated by the airway, cavities in PMEC could be observed, especially in the peripheral type. In this study, 2 cases of peripheral types in PMEC confirmed this owing to cavity formation.

Calcification was observed in 2 cases of PMEC, which showed relatively low incidence. However, in any case of PACC, calcification was not detected in any case of PACC, as calcification easily occurs in substances, which are rich in mucus materials. In literature reported by Tae SK, PMEC demonstrated punctuate calcification, which may be seen in up to $50 \%$ of lesions, especially in solitary nodules (6).

\section{Enhancement}

In some studies, the solid part of PMEC usually showed moderate or severe enhancement, but the solid part of PACC usually showed slight enhancement $(5,6)$. Our study showed a similar result, wherein we reported that $83.3 \%$ cases had moderate or severe enhancement in PMEC, and $80 \%$ cases had slight enhancement in PACC.

\section{Infiltration of lesion and metastasis of lymph nodes}

In our study, more infiltration of the mediastinal fat, thyroid, and esophagus were observed in PACC than the infiltration of the surrounding tissue seen in PMEC. Spizarny et al. reported that PACC had a predilection for the more proximal airways (i.e., distal trachea to main bronchi), as well as a propensity for extratracheal extension (16). However, this was in contrast to PMEC, which characteristically affected lobar or segmental bronchi and extended within the airways. Therefore, the infiltration for extratracheal extension was more common in PACC. However, some studies reported that PMEC grow slowly but showed progressive aggression (17), which needed further research and follow-up.

Similar to our results, Zhu also reported that both PMEC and PACC could show metastases of hilar and/or mediastinal lymph nodes (18). Further, this situation usually occurred in high-grade tumor lesions. In the series reported by Vadasz and Egervary (19), only high-grade tumors developed lymph node metastasis. The metastases in highgrade tumors were more common than those in low-grade tumors.

\section{Treatment and prognosis}

Both PMEC and PACC are low-grade malignancies, which are mainly treated by surgical resection. The utility of chemotherapy and radiotherapy in these cases remains controversial. When complete resection is not possible, adjuvant therapy should be administered (20).

The current literature indicated that the MECT1MAML2 fusion gene was common in PMEC and was specific to this tumor (21). MAML2 rearrangement was much more common in low-grade $(73.9 \%)$ than high-grade $(18.8 \%)$ cases. The 5 -year overall survival was also better in the MAML2 rearrangement-positive group (94.7\% vs. $64.6 \%$ in patients without MAML2 rearrangement). Thus, MAML2 rearrangement may signal a better prognosis in cases of PMEC.

\section{Conclusions}

PMEC and PACC have different CT features in various locations in the airway, which constitute crucial knowledge for radiologists and can be used as valuable imaging indicators for accurate diagnosis. PMEC is usually located in the lobar or segmental bronchi, usually accompanied by distal bronchial dilatation with mucoid impaction. PACC is usually located in trachea or main bronchi, with longitudinal extent greater than $3 \mathrm{~cm}$ and perimeter infiltration more than $1 / 2$ perimeter of luminal wall. Patchy areas of low density are more usually observed in PMEC than in PACC. The solid part of PMEC usually shows moderate or severe 
enhancement, but the solid part of PACC usually shows slight enhancement.

\section{Acknowledgements}

The authors would like to thank Xinchun Li and Yongxia Lei of Guangzhou Medical University.

Funding: This work was supported by the Natural Science Foundation (2015A030310473) of Guangdong Province.

\section{Footnote}

Conflicts of Interest: The authors have no conflicts of interest to declare.

\section{References}

1. Wang M, Ouyang SY, Sun PZ, et al. Pulmonary mucoepidermoid carcinoma in Chinese population: a clinicopathological and radiological analysis. Int J Clin Exp Pathol 2015;8:3001-7.

2. Jiang L, Li P, Xiao Z, et al. Prognostic factors of primary pulmonary mucoepidermoid carcinoma: a clinical and pathological analysis of 34 cases. Int J Clin Exp Pathol 2014;7:6792-9.

3. Macchiarini P. Primary tracheal tumours. Lancet Oncol 2006;7:83-91.

4. Stevic R, Milenkovic B. Tracheobronchial tumors. J Thorac Dis 2016;8:3401-13.

5. Elnayal A, Moran CA, Fox PS, et al. Primary Salivary Gland-Type Lung Cancer: Imaging and Clinical Predictors of Outcome. AJR Am J Roentgenol 2013;201:W57-63.

6. Kim TS, Lee KS, Han J, et al. Mucoepidermoid carcinoma of the tracheobronchial tree: radiographic and CT findings in 12 patients. Radiology 1999;212:643-8.

7. Xi JJ, Jiang W, Lu SH, et al. Primary pulmonary mucoepidermoid carcinoma: an analysis of 21 cases. World J Surg Oncol 2012;10:232.

8. Travis WD, Brambilla E, Kourad H, et al. Pathology and genetics of tumors of the lung, pleura, thymus and heart. In: Kleihues P, Sobin LH. editors. WHO Classification of Tumors 2nd edition. Lyon, France: IARC Press, 2004.

Cite this article as: $\mathrm{Li} \mathrm{X}, \mathrm{Yi} \mathrm{W}$, Zeng Q. CT features and differential diagnosis of primary pulmonary mucoepidermoid carcinoma and pulmonary adenoid cystic carcinoma. J Thorac Dis 2018;10(12):6501-6508. doi: 10.21037/jtd.2018.11.71
9. Wang S, Shi ML, Wu N, et al. Imaging findings of salivary gland-like neoplasms of the tracheobronchial tree. Chin J Radiol 2002;36:127-30.

10. K Bhatia, S Ellis. Unusual lung tumours: an illustrated review of CT features suggestive of this diagnosis. Cancer Imaging 2006;6:72-82.

11. Ngo AV, Walker CM, Chung JH, et al. Tumors and tumor like conditions of the large airways. AJR Am J Roentgenol 2013;201:301-13.

12. Fraser RS, Muller NL, Coleman N, Pare PD. Fraser and Pare's diagnosis of diseases of the chest. 4th ed. Philadelphia: Saunders, 1999.

13. Kwak SH, Lee KS, Chung MJ, et al. Adenoid cystic carcinoma of the airways: helical CT and histopathologic correlation. AJR Am J Roentgenol 2004;183:277-81.

14. Song Z, Liu Z, Zhang Y. Primary tracheobronchial mucoepidermoid carcinoma - a retrospective study of 32 cases patients. World J Surg Oncol 2013;11:62.

15. Molina JR, Aubry MC, Lewis JE, et al. Primary salivary gland-type lung cancer: spectrum of clinical presentation, histopathologic and prognostic factors. Cancer 2007;110:2253-9.

16. Spizarny DL, Shepard JA, McLoud TC, et al. CT of adenoid cystic carcinoma of the trachea. AJR Am J Roentgenol 1986;146:1129-32.

17. Shilo K, Foss RD, Franks TJ, et al. Pulmonary mucoepidermoid carcinoma with prominent tumor associated lymphoid proliferation. Am J Surg Pathol 2005;29:407-11.

18. Zhu F, Liu Z, Hou Y, et al. Primary salivary gland-type lung cancer: clinicopathological analysis of 88 cases from China. J Thorac Oncol 2013;8:1578-84.

19. Vadasz P, Egervary M. Mucoepidermoid bronchial tumors: a review of 34 operated cases. Eur J Cardiothorac Surg 2000;17:566-9.

20. Pandey D, Garg PK, Jakhetiya A, et al. Surgical experience of primary salivary gland tumors of lung: A case series. Int J Surg 2015;21:92-6.

21. Li X, Guo ZB, Liu JH, et al. Clinicopathological characteristics and molecular analysis of primary pulmonary mucoepidermoid carcinoma: Case report and literature review. Thorac Cancer 2018;9:316-23. 